\title{
BIOSSÓLIDO NA COMPOSIÇÃO DE SUBSTRATO PARA A PRODUÇÃO DE MUDAS DE Tectona grandis
}

\author{
Marcos Vinicius Winckler Caldeira ${ }^{1}$, William Macedo Delarmelina ${ }^{2}$, Sara Gütler Lübe ${ }^{3}$, \\ Daniele Rodrigues Gomes ${ }^{3}$, Elzimar de Oliveira Gonçalves ${ }^{4}$, Aloísio Favoreto Alves ${ }^{5}$ \\ ${ }^{1}$ Eng. Florestal, Dr., Depto. de Engenharia Florestal, UFES, Alegre, ES, Brasil - caldeiaramv@pq.cnpq.br \\ ${ }^{2}$ Acadêmico de Eng. Florestal, UFES, Alegre, ES, Brasil - williamdm@ hotmail.com \\ ${ }^{3}$ Acadêmica de Eng. Florestal, UFES, Alegre, ES, Brasil - saralube@ hotmail.com; daninha-gomes@ hotmail.com \\ ${ }^{4}$ Eng $^{\mathrm{a}}$. Florestal, Dr ${ }^{\mathrm{a}} .$, Depto. de Engenharia Florestal, UFES, Alegre, ES, Brasil - elzimarog@ yahoo.com.br \\ ${ }^{5}$ Acadêmico de Agronomia, UFES, Alegre, ES, Brasil - aloisiofavoreto@gmail.com
}

Recebido para publicação: 28/10/2010 - Aceito para publicação: 17/09/2011

\begin{abstract}
Resumo
Este estudo teve por objetivo avaliar o efeito do biossólido como componente do substrato para produção de mudas de Tectona grandis Linn. F. O experimento foi realizado em seis tratamentos com cinco repetições cada, sendo cada repetição constituída da média de cinco mudas. Os tratamentos foram formulados com as seguintes proporções de biossólido:substrato comercial (v:v); T1-20:80; T2-40:60; T3-60:40; T4-80:20; T5-100:0 e T6-0:100. Após 120 dias, as mudas foram coletadas para determinação das seguintes variáveis morfológicas: diâmetro do coleto, altura da parte aérea, relação entre a altura da parte aérea e o diâmetro do coleto, massa seca da parte aérea, sistema radicular e massa seca total, relação entre a matéria seca da parte aérea e a matéria seca do sistema radicular e índice de qualidade de Dickson. O biossólido favoreceu o crescimento das mudas de Tectona grandis. O máximo crescimento das mudas foi verificado quando se utilizou $60 \%$ a $100 \%$ de biossólido associado ao substrato comercial, o qual proporcionou melhores crescimentos dos parâmetros altura da parte aérea, diâmetro do coleto, massa seca total e índice de qualidade de Dickson.

Palavras-chave: Lodo de esgoto; teca; parâmetros morfológicos.
\end{abstract}

\begin{abstract}
Biosolids in composition of substrate for Tectona grandis Linn. F. seedlings production. This study aimed to evaluate the effect of biosolids as component of substrate for seedlings of Tectona grandis. The experiment was improved in six treatments with five repetitions each, such repetitions consisted of an average of five seedlings. The treatments were formulated with the following proportions of biosolids: commercial substrate (v:v); T1-20:80; T2-40:60; T3-60:40; T4-80:20; T5-100:0 and T6$0: 100$. After a hundred twenty days the seedlings were collected for determination of the following morphological variables: stem diameter, plant height, ratio between plant height and stem diameter, dry mass of shoot, dry mass of roots, the total dry mass, dry mass ratio of shoot/root dry mass and the quality index of Dickson. Biosolids favored seedlings of Tectona grandis growth. Maximum seedling development was observed when it was used $60 \%$ to $100 \%$ of biosolids associated with the commercial substrate, which provided better development for parameters as plant height, stem diameter, total dry mass and Dickson quality index.

Keywords: Sewage sludge; teca; morphological parameters.
\end{abstract}

\section{INTRODUÇÃO}

A Estação de Tratamento de Esgoto (ETE) é o conjunto de instalações e equipamentos destinados à realização do tratamento de esgotos, sejam eles domésticos ou industriais (SILVA et al., 2004). Os tratamentos realizados na ETE resultam na geração, entre outros produtos, de um resíduo sólido, representado pelo acúmulo de sólidos suspensos, chamado de Lodo de Esgoto (LE) ou biossólido.

O biossólido contém matéria orgânica, macro e micronutrientes que exercem um papel fundamental na produção agrícola e na manutenção da fertilidade do solo. Além disso, a matéria orgânica contida nos biossólidos pode aumentar o conteúdo de húmus, que melhora a capacidade de 
armazenamento e de infiltração da água no solo, aumentando a resistência dos agregados e reduzindo a erosão (BETTIOL; CAMARGO, 2000). Entretanto, a aplicação desse lodo no solo deve ser planejada, para que não ocorram problemas posteriores, visto que podem provocar alterações nas propriedades físicas, químicas e biológicas do solo, além da possibilidade de contaminação por agentes patogênicos (BOWER; IDELOVITCH, 1987) e metais pesados, no caso do material de origem industrial. A aplicação direta no solo de lodo "fresco" pode não ser a melhor forma, pois a atividade microbiana pode retirar todo o oxigênio do solo, tornando-o impróprio para cultivo.

O Brasil possui uma crescente demanda por produtos florestais, implicando o aumento do consumo de fertilizantes minerais no setor florestal, ocasionado pela alta degradação dos solos destinados a essa atividade. Com base nisso, a utilização de fertilizantes orgânicos, tal como o biossólido, poderá contribuir para redução do uso de fertilizantes minerais. Além disso, quando utilizado como substrato para a produção de mudas, se torna uma alternativa para a redução de custos, tendo em vista sua disponibilidade e facilidade de aquisição.

Segundo Gonçalves et al. (2000), deve-se dar prioridade de uso para os substratos que constituem resíduos industriais ou urbanos, pois, além de diminuir o problema ambiental, geralmente são uma garantia de fornecimento de matéria-prima a longo prazo e baixo custo (CALDEIRA et al., 2000a; CALDEIRA et al., 2000b; CALDEIRA et al., 2005; CALDEIRA et al., 2007; CALDEIRA et al., 2008a; CALDEIRA et al., 2008b). Devido a isso, vêm sendo realizados estudos para que esse produto originado de estações de tratamento de esgoto passe a ser utilizado de forma adequada para fins agrícolas e florestais, já que esse material possui um potencial fertilizante.

Vários substratos, em sua constituição original e combinada, são utilizados atualmente para a produção de mudas de espécies florestais (CALDEIRA et al., 2008a; CALDEIRA et al., 2008b; TRAZZI, 2011; KRATZ, 2011). Existem características que são consideradas essenciais para a obtenção de um bom substrato (GONÇALVES, 1981; CARNEIRO, 1995), entre as quais podem-se citar: a) boa estrutura e consistência, de forma a sustentar, sem grandes acomodações ou movimentações, as sementes e estacas durante a germinação e o enraizamento; b) substrato suficientemente poroso para permitir pronta drenagem do excesso de água durante as irrigações e chuvas, mantendo uma adequada aeração junto ao sistema radicial; c) boa capacidade de retenção de água, de modo a evitar as irrigações muito frequentes; d) não devem contrair ou expandir excessivamente com as oscilações de umidade; e) devem ser isentos de substâncias tóxicas, inóculos de doenças, plantas invasoras, insetos e sais em excesso; f) devem ser prontamente disponíveis em quantidades adequadas e custos economicamente viáveis; g) devem ser bem padronizados, com características físicas e químicas pouco variáveis de lote para lote; h) devem apresentar boa homogeneidade de partículas, bem como poucas partículas inertes, sobretudo as grandes, que tomam muito espaço sem nenhuma contribuição para a capacidade de agregação e retenção de água e nutrientes, principalmente para uso em recipientes com pequeno volume; i) ter boa capacidade de campo e capacidade de troca catiônica. Rosa Jr. et al. (1998) explicam que os substratos para a produção de mudas podem ser definidos como sendo o meio adequado para sua sustentação e retenção de quantidades suficientes e necessárias de água, oxigênio e nutrientes, além de oferecer $\mathrm{pH}$ compatível, ausência de elementos químicos em níveis tóxicos e condutividade elétrica adequada. A fase sólida do substrato deve ser constituída por uma mistura de partículas minerais e orgânicas.

O estudo da proporção de biossólido no substrato para produção de mudas é importante, já que ele poderá ser fonte de nutrientes e atuará diretamente sobre o crescimento das plantas. Portanto, em decorrência do arranjo quantitativo e qualitativo dos materiais minerais e orgânicos empregados, as mudas serão influenciadas pelo suprimento de nutrientes, água disponível e oxigênio (TRIGUEIRO; GUERRINI, 2003; ROSA JR. et al., 1998; TRAZZI, 2011; KRATZ, 2011).

A espécie Tectona grandis Linn. F. (teca), cujas sinonímias botânicas são Tectona theka Lour e Theka grandis (L.F.) Lam., é originária do continente asiático, estando sua área de ocorrência confinada entre Florestas Úmidas e Decídua Árida Mista. Essa espécie foi introduzida na região Norte do Brasil em 1994, com a finalidade de cumprir a reposição florestal obrigatória em atendimento à legislação ambiental vigente (FIGUEIREDO, 2005), tendo ultimamente conquistado espaço entre as principais culturas florestais do país. Sua madeira nobre, de excelente qualidade, é valorizada pela beleza, resistência e durabilidade. Tem grande procura no mercado mundial, onde pode alcançar preços até três vezes superiores ao do mogno, sendo utilizada na produção de móveis, esquadrias de alto padrão, embarcações e decoração (MACEDO et al., 1999). 
O objetivo desse estudo foi avaliar a viabilidade de produção de mudas de Tectona grandis Linn. F. utilizando doses crescentes do biossólido como substrato e como fonte de nutrientes no crescimento das mudas.

\section{MATERIAL E MÉTODOS}

O experimento utilizando doses crescentes de biossólido como substrato foi realizado com a espécie Tectona grandis Linn. F. (teca), em casa de sombra situada no Viveiro Florestal do Centro de Ciências Agrárias da Universidade Federal do Espírito Santo, localizado na Rodovia Cachoeiro-Alegre, km 06 (Área Experimental I) no município de Alegre (ES), durante o ano de 2009.

O clima predominante é quente e úmido no verão, com inverno seco e precipitação anual média de $1200 \mathrm{~mm}$ e temperatura média anual de $23^{\circ} \mathrm{C}$ (ESPÍRITO SANTO, 1994).

As sementes (frutos) utilizadas foram obtidas a partir de doação da empresa Floresteca S/A. Fezse a quebra de dormência das mesmas, deixando-as 12 horas em água corrente no período noturno e 12 horas exposta ao ar e no sol durante três dias consecutivos. Após o tratamento de quebra de dormência, foi realizada a semeadura em sementeiras constituídas de uma camada de brita e outra, em maior proporção, de areia, cobertas com uma camada fina do mesmo substrato (areia), sendo irrigadas por aspersão três vezes ao dia.

As plântulas permaneceram por um mês nas sementeiras desde a semeadura e após atingirem $5,0 \mathrm{~cm}$ de altura e apresentarem, no mínimo, dois pares de folhas foram repicadas para tubetes com capacidade de $120 \mathrm{~cm}^{3}$ de substrato.

O presente estudo foi montado em um delineamento inteiramente casualizado (DIC) com seis tratamentos e cinco repetições, sendo que para cada repetição foi considerada a média de cinco mudas escolhidas aleatoriamente de um total de 25 mudas por tratamento. As proporções de biossólido:substrato comercial (v:v), contidas em cada tratamento foram; T1-20:80; T2-40:60; T3-60:40; T4-80:20; T5-100:0 e T6-0:100.

Para garantir a capacidade nutricional das mudas em todos os tratamentos, foram adicionados 25 gramas de fertilizante mineral N-P-K (04-16-04) em cada tratamento, e após três meses de repicagem foi feita uma adubação foliar nitrogenada com dosagem de 300 gramas de ureia dissolvida em 20 litros de água, com adição de $50 \mathrm{ml}$ dessa solução por tratamento contendo 25 mudas. O suprimento de água foi garantido por irrigação automática três vezes por dia. O substrato comercial utilizado no experimento foi formado por $60 \%$ de composto de casca de pinus, $15 \%$ de vermiculita e $25 \%$ de húmus mais terra vegetal.

O biossólido utilizado no experimento foi doado pela Foz do Brasil S.A. (ETE de Cachoeiro de Itapemirim, ES). Na tabela 1, observa-se que as quantidades de metais pesados poluentes presentes no biossólido são baixas, e, de acordo com a resolução CONAMA no 375/2006, esse material está apto para uso em ambientes agrícolas.

Tabela 1. Teores $\left(\mathrm{mg} \cdot \mathrm{dm}^{-3}\right)$ de metais pesados poluentes no biossólido doado pela Foz do Brasil S.A. na estação de tratamento de esgoto de Cachoeiro de Itapemirim, ES.

Table 1. Content $\left(\mathrm{mg} \mathrm{dm}^{-3}\right)$ of heavy metal pollutants in biosolids donated by Foz do Brasil S.A. from Itapemirim-ES treatment plant sewer.

\begin{tabular}{lcc}
\hline Parâmetros & Resultados analíticos $^{\mathbf{1}}$ & Resolução CONAMA - 375/2006 $^{\mathrm{Ar}}$ \\
$\mathrm{Ba}$ & $<0,5$ & 41 \\
$\mathrm{Cd}$ & 156 & 1300 \\
$\mathrm{~Pb}$ & $<0,053$ & 300 \\
$\mathrm{Cu}$ & 29 & 1500 \\
$\mathrm{Cr}$ & 98 & 1000 \\
$\mathrm{Mb}$ & 26 & 50 \\
$\mathrm{Ni}$ & 3,5 & 420 \\
$\mathrm{Se}$ & 11 & 100 \\
\hline
\end{tabular}

${ }^{1}$ Análise realizada pela Foz do Brasil S.A.

Os substratos que compõem os tratamentos foram analisados quimicamente antes da montagem do experimento, segundo a metodologia de Tedesco et al. (1995) (Tabela 2). As análises foram realizadas 
no Laboratório de Análises de Fertilizantes, Águas, Minérios, Resíduos, Solos e Plantas (LAFARSOL) do Centro de Ciências Agrárias da Universidade Federal do Espírito Santo.

Tabela 2. Teores totais de macro e micronutrientes, matéria orgânica (MO) e relação $\mathrm{C} / \mathrm{N}$ dos substratos utilizados na formulação dos tratamentos.

Table 2. Total content of macro and micronutrients, organic matter $(\mathrm{OM})$ and $\mathrm{C} / \mathrm{N}$ ratio of substrates used in the formulation of treatments.

\begin{tabular}{|c|c|c|c|c|c|c|c|c|c|c|c|c|c|}
\hline \multirow{2}{*}{ Substrato } & $\mathbf{N}$ & $\mathbf{P}$ & $\mathbf{K}$ & $\mathbf{C a}$ & Mg & $\mathbf{S}$ & Zn & $\mathbf{F e}$ & Mn & $\mathbf{C u}$ & B & \multirow{2}{*}{$\begin{array}{c}\text { MO } \\
\text { g.kg }^{-1}\end{array}$} & \multirow{2}{*}{$\mathbf{C} / \mathbf{N}$} \\
\hline & \multicolumn{6}{|c|}{ g.kg ${ }^{-1}$} & \multicolumn{5}{|c|}{ mg.kg ${ }^{-1}$} & & \\
\hline SC & 8,8 & 1,6 & 1,2 & 8,3 & 4,1 & 0,06 & 44 & 9200 & 199 & 10 & 14 & 145,9 & 9,6 \\
\hline $\mathrm{BIO}$ & 13,3 & 2,5 & 0,8 & 8,9 & 2,4 & 0,14 & 231 & 17480 & 157 & 53 & 8 & 65,5 & 2,9 \\
\hline
\end{tabular}

SC: substrato comercial; BIO: Biossólido.

As mudas de Tectona grandis foram coletadas 120 dias após a repicagem, para a determinação do diâmetro do coleto $(\mathrm{D})$, altura da parte aérea $(\mathrm{H})$, relação entre a altura da parte aérea, diâmetro do coleto (RHD), Massa Seca da Parte Aérea (MSPA), Massa Seca do Sistema Radicular (MSR), Massa Seca Total (MST), relação entre a massa seca da parte aérea e massa seca do sistema radicular (RMPAR) e Índice de Qualidade de Dickson (IQD).

O diâmetro do coleto foi obtido com paquímetro digital e a altura determinada a partir do nível do substrato até a inserção da última folha, com auxílio de uma régua graduada em milímetros. A quantificação do peso da matéria seca para a obtenção da MSPA, MSR, MST, relação entre a massa seca da parte aérea e a RMPAR, foi feita através da pesagem das partes vegetais, após a secagem em estufa de circulação de ar forçada a $70{ }^{\circ} \mathrm{C}$ por um período de aproximadamente $72 \mathrm{~h}$. O Índice de Qualidade de Dickson foi obtido pela fórmula de Dickson et al. (1960):

$$
\mathrm{IQD}=\frac{\mathrm{MST}_{(\mathrm{g})}}{\mathrm{H}_{(\mathrm{cm})} / \mathrm{DC}_{(\mathrm{mm})}+\mathrm{MSPA}_{(\mathrm{g})} / \mathrm{MSR}_{(\mathrm{g})}}
$$

As variáveis coletadas foram submetidas à análise de variância (ANOVA) e as médias comparadas pelo teste de Tukey a 5\% de probabilidade.

\section{RESULTADOS E DISCUSSÃO}

Os resultados obtidos com a adição de biossólido na composição de substrato após 120 dias da repicagem são apresentados na tabela 3 .

Tabela 3. Características morfológicas de mudas de Tectona grandis produzidas em substratos contendo diferentes percentagens de biossólido, aos 120 dias de idade. Alegre, ES, 2009.

Table 3. Morphological characteristics of Tectona grandis seedlings, produced in substrates containing different percentages of sewage sludge at 120 days old. Alegre, ES, 2009.

\begin{tabular}{lcccccccc}
\hline Substrato & H $(\mathbf{c m})$ & $\mathbf{D}(\mathbf{m m})$ & RHD & MSPA $(\mathbf{g})$ & MSR(g) & MST $(\mathbf{g})$ & RMSPAR & IQD \\
\hline $20: 80$ & $8,1 \mathrm{~b}$ & $8,5 \mathrm{~b}$ & $0,94 \mathrm{bc}$ & $0,939 \mathrm{bc}$ & $1,159 \mathrm{c}$ & $2,098 \mathrm{~b}$ & 0,81 & $1,19 \mathrm{c}$ \\
$40: 60$ & $11,7 \mathrm{a}$ & $10,0 \mathrm{a}$ & $1,18 \mathrm{~b}$ & $1,526 \mathrm{ab}$ & $1,813 \mathrm{ab}$ & $3,340 \mathrm{a}$ & 0,83 & $1,66 \mathrm{abc}$ \\
$60: 40$ & $9,5 \mathrm{~b}$ & $8,9 \mathrm{ab}$ & $1,07 \mathrm{bc}$ & $1,622 \mathrm{a}$ & $1,529 \mathrm{bc}$ & $3,152 \mathrm{a}$ & 1,06 & $1,47 \mathrm{bc}$ \\
$80: 20$ & $8,1 \mathrm{~b}$ & $9,9 \mathrm{a}$ & $0,82 \mathrm{c}$ & $1,807 \mathrm{a}$ & $2,024 \mathrm{ab}$ & $3,832 \mathrm{a}$ & 0,93 & $2,23 \mathrm{a}$ \\
$100: 0$ & $9,0 \mathrm{~b}$ & $9,0 \mathrm{ab}$ & $0,98 \mathrm{bc}$ & $1,699 \mathrm{a}$ & $2,060 \mathrm{a}$ & $3,760 \mathrm{a}$ & 0,82 & $2,08 \mathrm{ab}$ \\
$0: 100$ & $5,6 \mathrm{c}$ & $3,5 \mathrm{c}$ & $1,60 \mathrm{a}$ & $0,568 \mathrm{c}$ & $0,379 \mathrm{~d}$ & $0,948 \mathrm{c}$ & 1,42 & $0,30 \mathrm{~d}$ \\
\hline $\mathrm{F}$ & $* *$ & $* *$ & $* *$ & $* *$ & $* *$ & $* *$ & $\mathrm{~ns}$ & $* *$ \\
$\mathrm{CV} \%$ & 9,88 & 6,85 & 12,21 & 19,4 & 15,68 & 14,6 & 38,61 & 18,15
\end{tabular}

Altura (H), diâmetro do coleto (DC), massa seca parte aérea (MSPA), massa seca radicular (MSR), massa seca total (MST), relação altura/diâmetro (RHD), relação massa seca da parte aérea/raiz (RPAR) e índice de qualidade de Dickson (IQD). Ns: não significativo $(\mathrm{P}>0,05)$; **significativo $(\mathrm{P}<0,01)$. Médias seguidas da mesma letra, na coluna, não diferem estatisticamente entre si pelo teste Tukey $(\mathrm{P}>0,05)$. Substrato: Biossólido:substrato comercial em volumes de mistura. 
Segundo Mexal e Lands (1990), a altura da parte aérea das mudas fornece uma excelente estimativa da predição do crescimento inicial da mudas no campo, sendo tecnicamente aceita como uma boa medida do potencial de desempenho das mudas. No estudo, a altura da parte aérea apresentou crescimento estatisticamente superior no tratamento com $40 \%$ de biossólido. Com base nas médias obtidas, verifica-se que o biossólido proporcionou efeito positivo em todos os tratamentos quando comparadas à testemunha (apenas substrato comercial). Resultados semelhantes foram obtidos por Nóbrega et al. (2007) avaliando a influência de doses de biossólido formuladas com Neossolo Quartzarênico e de Latossolo Vermelho-Amarelo em mudas de Schinus terebinthifolius Raddi. Os autores observaram uma tendência ao aumento desse parâmetro a partir da primeira dose de biossólido (20\%) adicionada ao substrato. Os autores ainda observam que, devido ao acréscimo de nutrientes com a adição do biossólido, as mudas atingiram a altura máxima estimada de $15,9 \mathrm{~cm}$ com a dose de $35 \%$, seguida de redução com doses maiores.

Para a característica diâmetro do coleto, os tratamentos T2 (40\% de biossólido) e T4 (80\% de biossólido) foram os que apresentaram melhores resultados, sendo que o tratamento $\mathrm{T} 2$ obteve a melhor média. Essa característica, em geral, é a mais observada para avaliar a capacidade de sobrevivência da muda no campo, sendo, assim, a mais indicada para auxiliar na definição das doses de fertilizantes a serem aplicadas, objetivando a avaliação da produção e qualidade de mudas arbóreas (CARNEIRO, 1995). Da mesma forma, Maas (2010), utilizando mudas de timburi (Enterolobium contortisiliquun (Vell.) Morong), verificou que o tratamento com $100 \%$ de substrato comercial apresentou dados inferiores aos tratamentos que utilizavam 15, 30, 45 e $60 \%$ de biossólido associados ao substrato comercial para o parâmetro diâmetro do coleto. Nesse sentido, o autor avalia a adição do biossólido como um efeito positivo em relação a essa variável, obtendo mudas com maior probabilidade de sobrevivência quando levadas a campo.

A relação entre altura e diâmetro do colo indica a qualidade da muda em qualquer fase do período de produção, devendo situar-se entre os limites de 5,4 a 8,1 (CARNEIRO, 1995). Nessa variável, o melhor resultado evidenciado foi no tratamento testemunha (100\% de substrato comercial), que apresentou maior valor. Todas as médias obtidas para essa característica foram abaixo dos limites propostos por Carneiro (1995), o que indica um elevado incremento do diâmetro não acompanhado pelo crescimento em altura das mudas. No entanto, essas mudas não devem ser desconsideradas, uma vez que a principal desvantagem desse método é que o sistema radicular não é avaliado (CARNEIRO, 1995). Cabe ressaltar que a relação pode variar em função da espécie, do tipo e proporção do substrato, do volume do recipiente, do manejo das mudas no viveiro e da idade em que a muda foi avaliada.

As proporções de biossólido:substrato comercial que evidenciaram maiores médias de massa seca da parte aérea foram 60:40, 80:20, 100:0. Com isso houve um contraste com a análise realizada por Gomes e Paiva (2006), para quem a relação entre os fatores que influenciam no crescimento em altura da parte aérea das mudas é também responsável pela matéria seca. Já no que se refere à massa seca de raiz, o substrato comercial promoveu menor produção de biomassa em relação aos tratamentos com biossólido. O contrário foi relatado por Trigueiro e Guerrine (2003), em que o substrato comercial proporcionou melhores resultados de massa seca de raiz para produção de mudas de Eucalyptus grandis.

A produção de matéria seca total foi inferior no tratamento T6, sem a utilização de biossólido, sendo que os tratamentos com 40,60,80 e 100\% de biossólido devidamente formulados com o substrato apresentaram resultados estatisticamente superiores. Infere-se que, em decorrência do aumento das doses de biossólido no substrato, houve aumento no aproveitamento dos nutrientes pela planta e consequente aumento na produção de MST, tendo a proporção de $80 \%$ biossólido a maior média.

Considerando que a relação massa seca da parte aérea/massa seca das raízes depende de dois parâmetros destrutivos para sua determinação, que os dados não permitem maiores conclusões e que é uma relação contraditória para o crescimento de mudas no campo (BURNETT, 1979), não deverá ser indicada como índice para determinação do padrão de qualidade de mudas de Eucalyptus grandis (GOMES et al., 2002) e provavelmente para outras espécies também. Não houve diferença significativa entre os tratamentos quando se avaliou a relação entre a massa seca da parte aérea e a massa seca do sistema radicular. Pode-se dizer que valores muito altos para esse parâmetro pode ser prejudiciais à muda, devido à tendência de desequilíbrio no crescimento e consequente tombamento das mudas e possíveis problemas no que se refere à absorção de água para a parte aérea. 
O IQD é apontado como bom indicador de qualidade de mudas, porque são utilizados para seu cálculo a robustez (relação H/DC) e o equilíbrio da distribuição da biomassa (relação MSPA/MSR) (FONSECA, 2000; CALDEIRA et al., 2005; CALDEIRA et al., 2007; TRAZZI, 2011). Com relação ao Índice de Qualidade de Dickson, as mudas do tratamento T4 (80\% de biossólido) obtiveram o melhor resultado final. Observa-se que os maiores IQD calculados apresentam no geral maiores valores de D, MSPA, MSRA e MST, o que implica melhor qualidade da muda. Com base nisso, verifica-se que mudas do tratamento testemunha (T6) apresentaram uma baixa distribuição da biomassa, acarretando um menor equilíbrio da planta e assim pressupondo a influência positiva do biossólido na qualidade das mudas de Tectona grandis.

É possível constatar que vários estudos na literatura mostram que o índice de qualidade de Dickson é uma característica variável (CALDEIRA et al., 2000a; CALDEIRA et al., 2000b; CALDEIRA et al., 2007; CALDEIRA et al., 2005; CALDEIRA et al., 2008a; CALDEIRA et al., 2008b; SAIDELLES et al., 2009; TRAZZI et al., 2010; KRATZ, 2011; TRAZZI, 2011). Nesse sentido, pode-se concluir que esse índice pode variar em função da espécie, do manejo das mudas no viveiro, do tipo e proporção do substrato, do volume do recipiente e, principalmente, de acordo com a idade em que a muda foi avaliada.

Com os resultados obtidos neste estudo, nota-se que os tratamentos nos quais foi empregado biossólido apresentaram crescimento superior ao tratamento com apenas substrato comercial na sua formulação (T6). O mesmo ocorreu com experimento conduzido por Faustino et al. (2005), em que avaliaram a viabilidade do uso de lodo de esgoto como componente do substrato para produção de mudas de Senna siamea Lam., sendo testadas as seguintes proporções de biossólido:solo (\%): 0:100\% (T1), 25:75 (T2), 50:50 (T3), 75:25 (T4) e uma mistura de 25\% de lodo de esgoto com 25\% de pó de coco (T5). $\mathrm{O}$ tratamento T5 foi o que apresentou o melhor crescimento das mudas, seguido daqueles com $75 \%$ (T4) e $50 \%$ (T3) de lodo.

\section{CONCLUSÕES}

- O uso de biossólido na composição do substrato para produção de mudas de Tectona grandis é uma alternativa viável para a disposição final desses resíduos, tendo em vista a economia de fertilizantes na produção das mudas, além do benefício ambiental.

- Todos os tratamentos que possuem biossólido em sua formulação proporcionaram melhor crescimento em altura, diâmetro de colo e Índice de Qualidade de Dickson.

- Para produção de Tectona grandis com um adequado padrão de qualidade, recomenda-se utilizar a adição de mais de $60 \%$ de biossólido associado ao substrato comercial.

\section{REFERÊNCIAS}

BETTIOL, W.; CAMARGO, O. A. Impacto ambiental do uso do lodo de esgoto. Jaguariuna, SP: Embrapa Meio Ambiente, 2000. 312 p.

BOWER, H.; IDELOVITCH, E. Quality requirements for irrigation with sewage water. Journal of Irrigation and Drainage Engineering, New York, v. 113, n. 4, p. 510 - 535, 1987.

BURNETT, A. N. New methods for measuring root growth capacity: their value in assessing lodgepole pine stock quality. Canadian Journal of Forest Research, Ottawa, v. 9, p. 63 - 67, 1979.

CALDEIRA, M. V. W.; BLUM, H.; BALBINOT, R.; LOMBARDI, K. C. Uso do resíduo do algodão no substrato para produção de mudas florestais. Revista Acadêmica: Ciências Agrárias e Ambientais, v. 6, p. 191 - 202. 2008a.

CALDEIRA, M. V. W.; ROSA, G. N.; FENILLI, T. A. B.; HARBS, R. M. P. Composto orgânico na produção de mudas de aroeira-vermelha. Scientia Agraria, v. 9, p. 27 - 33. 2008 b.

CALDEIRA, M. V. W.; SPATHELF, P.; BARICHELLO, L. R.; VOGEL, H. L. M.; SCHUMACHER, M. V. Effect of different doses of vermicompost on the growth of Apuleia leiocarpa (Vog) Macbr. seedlings. Revista Acadêmica: Ciências Agrárias e Ambientais, v. 3, p. 11 - 17, 2005. 
CALDEIRA, M. V. W.; MARCOLIN, M.; MORAES, E.; SCHAADT, S. S. Influência do resíduo da indústria do algodão na formulação de substrato para produção de mudas de Schinus terebinthifolius Raddi, Archontophoenix alexandrae Wendl. et Drude e Archontophoenix cunninghamiana Wendl. et Drude. Ambiência, Guarapuava, v. 3, p. 1 - 8, 2007.

CALDEIRA, M. V. W.; SCHUMACHER, M. V.; BARICHELLO, L. R.; VOGET, H. L. M.; OLIVEIRA, L. S. Crescimento de mudas de Eucalyptus saligna Smith em função de diferentes doses de vermicomposto. Revista Floresta, Curitiba, v. 28, n. 1/2, p. 19 - 30, 2000a.

CALDEIRA, M. V. W.; SCHUMACHER, M. V.; TEDESCO. N. Crescimento de mudas de Acacia mearnsii em função de diferentes doses de vermicomposto. Scientia Forestalis, Piracicaba, n. 57, p. 161 - 170, 2000b.

CARNEIRO, J. G. A. Produção e controle de qualidade de mudas florestais. Universidade Federal do Paraná, Curitiba, 451 p. 1995.

DICKSON, A.; LEAF, A. L.; HOSNER, J. F. Quality appraisal of while spruce and white pine seedling stock in nurseries. Forestry Chronicle, v. 36, p. 11 - 13, 1960.

ESPÍRITO SANTO. Secretaria de Estado de Ações Estratégicas e Planejamento. Informações municipais do Estado do Espírito Santo, 1994. Vitória: Departamento Estadual de Estatística, 1994. v. $1.803 \mathrm{p}$.

FAUSTINO, R.; KATO, M. T.; FLORÊNCIO, L. GAVAZZA, S. Lodo de esgoto como substrato para a produção de mudas de Senna siamea Lam. Revista Brasileira de Engenharia Agrícola e Ambiental, Campina Grande, v. 9, p. 278 - 282, 2005.

FIGUEIREDO, E. O. Teca (Tectona grandis L.f.): produção de mudas tipo toco. Embrapa - Acre, Rio Branco, Acre, 22 p. 2005. (Documentos, 101).

FONSECA, E. P. Padrão de qualidade de mudas de Trema micrantha (L.) Blume., Cedrela fissilis Vell. E Aspidosperma polyneuron Müll. Arg. produzidas sob diferentes períodos de sombreamento. 2000. 113 p. Tese (Doutorado em Agronomia) - Universidade Estadual Paulista, Jaboticabal, 2000.

GOMES, J. M.; COUTO, L.; LEITE, H. G.; XAVIER, A.; GARCIA, S. L. R. Parâmetros morfológicos na avaliação da qualidade de mudas de Eucalyptus grandis. Revista Árvore, Viçosa, v. 26, n. 6, p. 655 $664,2002$.

GOMES, J. M.; PAIVA, H. N. Viveiros florestais: propagação sexuada. 3. ed. Viçosa: UFV, 2006.

GONÇALVES, A. N. Aspectos fisiológicos da multiplicação vegetativa. In: Seminário sobre propagação vegetativa, Brasília, 1981. 8 p.

GONÇALVES, J. L. M.; SANTARELLI, E. G.; MORAES NETO, S. P.; MANARA, M. P. Produção de mudas de espécies nativas: substrato, nutrição, sombreamento e fertilização. In: GONÇALVES, J. L. M.; BENEDETTI, V. (ed.) Nutrição e fertilização florestal. Piracicaba: Instituto de Pesquisas e Estudos Florestais, 2000. p. 309 - 350.

KRATZ, D. Substratos renováveis na produção de mudas de Eucalyptus benthamii Maiden et Cambage e Mimosa scabrella Benth. 2011. 121 f. il. Dissertação (Mestrado em Ciências Florestais) Universidade Federal do Paraná, Curitiba.

MAAS, K. D. B. Biossólido como substrato na produção de mudas de timburi. 46 f. Dissertação (Mestrado em Ciências Florestais e Ambientais) - Universidade Federal de Mato Grosso, Mato Grosso, 2010. Cuiabá, 2010.

MACEDO, R. L. G.; GOMES, J. E.; TSUKAMOTO FILHO, A. de A. Análise preliminares sobre o crescimento e fenologia de Tectonea grandis (teca), inplantada em parcelas de observação na região de Lavras, MG. In. SIMPÓSIO INTERNACIONAL SOBRE ECOSSISTEMAS FLORESTAIS, 5, Curitiba, 1999. Anais... Rio de Janeiro. Biosfera, 1999. 4 p. (CD ROM-BIO 1200). 
MEXAL, J. L.; LANDS, T. D. Target seedling concepts: height and diameter. In: TARGET SEEDLING SYMPOSIUM, MEETING OF THE WESTERN FOREST NURSERY ASSOCIATIONS, GENERAL TECHNICAL REPORT RM-200, 1990, Roseburg. Proceedings... Fort. Collins: United States Departament of Agriculture, Forest Service, 1990. p. 17 - 35.

NÓBREGA, R. S. A.; VILAS BOAS, R. C.; NÓBREGA, J. C. A.; PAULA, A. M.; MOREIRA, F. M. S. Utilização de biossólido no crescimento inicial de mudas de aroeira (Schinus terebynthifolius/Raddi). Revista Árvore, v. 31, n. 2. Viçosa, 2007.

ROSA JÚNIOR, E. J.; DANIEL, O.; VITORINO, A. C. T.; SANTOS FILHO, V. C. Efeito de diferentes substratos sobre o desenvolvimento de mudas de Eucalyptus grandis Hill, em tubetes. Ciência Agronômica, Ceará, p. 18 - 22, 1998.

SAIDELLES, F. L. F.; CALDEIRA, M. V. W.; SCHIRMER, W. N.; SPERANDIO, H. V. Casca de arroz carbonizada como substrato para produção de mudas de tamboril-da-mata e garapeira. Semina: Ciências Agrárias, v. 30, p. 1173 - 1186. 2009.

SILVA, W. T. L de.; NOVAES, A. P. de.; MARTIN NETO, L.; MILORI, D. M. B. P.; SIMÕES, M. L.; HANEDA, R. N.; FIALHO, L. L.; LEONELLI, F. C. V. Método de aproveitamento de biossólido proveniente de lodo de esgoto residencial através de processo de compostagem seguido de biodigestão anaeróbia. Relatório Técnico. São Carlos, SP: Embrapa Instrumentação Agropecuária, 2004.

TEDESCO, M. J.; GIANELlO, C.; BISSANI, C. A.; BOHNEN, H.; VOLKWEISS, S. J. Análises de solos, plantas e outros materiais. 2. ed., Porto Alegre: UFRGS, 1995. (Boletim Técnico, 5).

TRAZZI, P. A. Substratos renováveis na produção de mudas de Tectona grandis Linn F. 2011. 84 f. Dissertação (Mestrado em Ciências Florestais) - Universidade Federal do Espírito Santo, Alegre.

TRAZZI, P. A.; CALDEIRA, M. V. W.; COLOMBI, R. Avaliação de mudas de Tecoma stans utilizando biossólido e resíduo orgânico. Revista de Agricultura, Piracicaba, v. 85, p. 218 - 226. 2010.

TRIGUEIRO, R. de M.; GUERRINI, I. A. Uso de biossólido como substrato para produção de mudas de eucalipto. Scientia Forestalis, Piracicaba-SP, 2003, p. 150 - 162. 